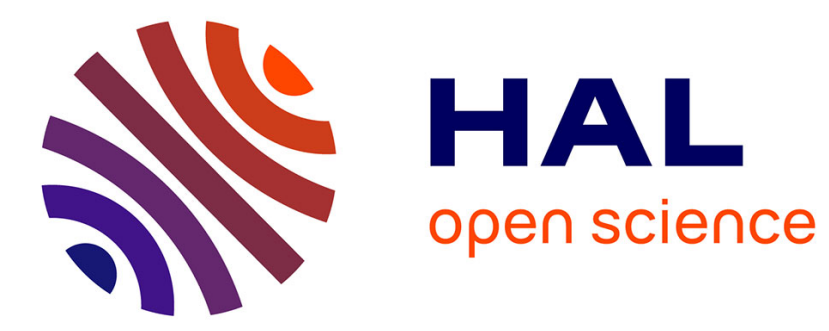

\title{
Imperfect supercritical bifurcation in a three-dimensional turbulent wake
}

Olivier Cadot, Antoine Evrard, Luc R. Pastur

\section{To cite this version:}

Olivier Cadot, Antoine Evrard, Luc R. Pastur. Imperfect supercritical bifurcation in a threedimensional turbulent wake. Physical Review Online Archive (PROLA), 2015, pp.063005. 10.1103/PhysRevE.91.063005 . hal-01164472

HAL Id: hal-01164472

https://hal-ensta-paris.archives-ouvertes.fr/hal-01164472

Submitted on 17 Jun 2015

HAL is a multi-disciplinary open access archive for the deposit and dissemination of scientific research documents, whether they are published or not. The documents may come from teaching and research institutions in France or abroad, or from public or private research centers.
L'archive ouverte pluridisciplinaire HAL, est destinée au dépôt et à la diffusion de documents scientifiques de niveau recherche, publiés ou non, émanant des établissements d'enseignement et de recherche français ou étrangers, des laboratoires publics ou privés. 


\title{
Imperfect supercritical bifurcation in a 3D turbulent wake
}

\author{
Olivier Cadot and Antoine Evrard \\ IMSIA-ENSTA ParisTech, CNRS, CEA, EDF, Université Paris-Saclay, Palaiseau, France. \\ Luc Pastur \\ LIMSI-Univ Paris Sud, CNRS, Université Paris-Saclay, Orsay, France.
}

(Dated: May 28, 2015)

\begin{abstract}
The turbulent wake of a square-back body exhibits a strong bi-modal behavior. The wake randomly undergoes symmetry breaking reversals between two mirror asymmetric steady modes (Reflectional Symmetry Breaking, $R S B$ modes). The characteristic time for reversals is about 2 or 3 orders of magnitude larger than the natural time for vortex shedding. Studying the effects of the proximity of a ground wall together with the Reynolds number, it is shown that the bi-modal behavior is the result of an imperfect pitchfork bifurcation. The $R S B$ modes correspond to the two stable bifurcated branches resulting from an instability of the stable symmetric wake. An attempt to stabilize the unstable symmetric wake is investigated using a passive control technique. Although the controlled wake still exhibits strong fluctuations, the bi-modal behavior is suppressed, and the drag reduced. This promising experiment indicates the possible existence of an unstable solution branch corresponding to a reflectional symmetry preserved $(R S P)$ mode. This work is encouraging to develop control strategy based on a stabilization of this $R S P$ mode to reduce mean drag and lateral force fluctuations.
\end{abstract}

PACS numbers: 47.20.Ky,47.15.Fe,47.27.Cn,47.27.wb

\section{INTRODUCTION}

In the Navier-Stokes equations, symmetry breaking and bifurcations are the key ingredients for the laminar to turbulent transition. They appear in the flow solutions as the control parameter (i.e. the Reynolds number) is increased. Normally, the strongest modifications in the flow appear during the lowest part of the transitional range of Reynolds numbers. The highest part of the transitional range is reached when all the symmetries are restored in the statistical sense [1]. However, there are some cases for which such high Reynolds number flows may undergo large scale symmetry breaking.

One of the most spectacular occurs during the drag crisis transitions of smooth bluff bodies [2]. In the critical Reynolds number regime of a circular cylinder $\left(10^{5}<R e<5 \times 10^{5}\right)$, the laminar to turbulent transition of the free boundary layers is accompanied by the occurrence of asymmetric wake flow states $[3,4]$ producing a non zero mean lift. They are commonly called one bubble and two bubbles transitions corresponding respectively to the reattachment on one side and both sides of the cylinder. From global force $[5,6]$ and local pressure [7] measurements, the existence of bistable behaviors in this critical range has been evidenced through hysteretic discontinuities in the Strouhal - Reynolds number relationship. Schewe [5] associated each discontinuity with a subcritical bifurcation during which the energy of the force fluctuations is dominated by the contribution of a wide low frequency domain [5-7]. This domain belongs to lower frequencies than the nearly extinguished frequencies of the Kármán global modes. Recently, the examination of pressure time series measured on the cylinder $[8,9]$ revealed that reattachments might switch from one side to the other unpredictably in time during the drag crisis fluctuations. Further analyses by Cadot et al. [10] confirmed that the strong fluctuations were the consequence of the random exploration of few identified asymmetric and symmetric metastable states.

Another case of large scale symmetry breaking was evidenced more recently in a totally different flow configuration [11-15]; the von-Kármán swirling flow geometry. It is basically a closed turbulent flow forced between two counter-rotating stirrers facing each other in a cylindrical tank. The transition that occurs around $\mathrm{Re}=10^{4}$, consists in a bifurcation of the basic symmetric flow topology toward two other flow topologies which break the symmetry of the driving geometry $[11,13]$. In $[12,14,15]$, random switching between two metastable symmetrybreaking states are observed. In that case, the symmetry is restored in a statistical sense, but a state remains observable over durations much larger than the timescale of the turbulence large scale. Another difference with the drag crisis case, is that the symmetry breaking modes in the von-Kármán swirling flow geometry are also observable in the laminar regime during the transition scenario to turbulence $[16,17]$ and the symmetry breaking in the turbulent regime might be reminiscent of these modes.

Even more recently, the three-dimensional wake of a generic square-back body has been shown to switch randomly between two asymmetric metastable states over a wide range of Reynolds numbers from 300 to $10^{7}$ [18-20]. Their observation in the laminar regime [18] excludes the possibility of an origin associated with turbulent boundary layer reattachment as for those present during the drag crisis transition. The authors referred to this as a bistable behavior of the wake, unpredictable in time with a long time dynamics, 2 or 3 orders of magnitude larger than the time dynamics for vortex shedding. The 
metastable states break the reflectional symmetry of the set-up, and will be referred to in the following as $R S B$ modes (Reflectional Symmetry Breaking modes). On the theoretical background, $R S B$ modes of three dimensional wakes are actually known to be bifurcated states of the basic symmetric state observed in the laminar regime (see the stability analysis of Pier [21] for a sphere or a disk and very recently, Marquet and Larsson [22] for rectangular plates of different aspect ratio). In the work of Grandemange et al. [20], the bistability was found to be inhibited when the body was approaching the ground wall at a Reynolds number of $4.5 \times 10^{4}$ leading to a high Reynolds number transition.

The present work aims at characterizing this transition in the turbulent wake using independently two control parameters: the ground clearance $C$ (distance from the ground wall to the body), and the Reynolds number Re. A global quantity giving a topological indicator of the wake will be used as the order parameter of the transition. The questions we would like to address are the following. Does the turbulent wake flow have an unstable symmetric solution when it explores randomly the metastable $R S B$ modes ? The answer might have relevant applications for flow control strategies at industrial scale (i.e. large Reynolds number flow). Secondly from a more general perspective, what are the similarities between this transition and those of the von-Kármán swirling flow geometry?

The article is organized as follow. Section II (Experimental set-up) is in two parts and describes the geometry and the measurements. The Results section III is presented in five parts. Part III A defines and characterizes the global quantity that is used as the order parameter to describe the transition. Part III B shows that the transition does not depend on the yaw angle of the body. Part III C evidences the existence of a pitchfork bifurcation and sets the phase diagram of the bifurcation vs. ground clearance and Reynolds number. In Part III D we attempt to stabilize the turbulent symmetric wake flow and part III E illustrates the main result with some flow visualizations in a water tunnel at a comparable Reynolds number. Finally, conclusive remarks end the paper in section IV.

\section{EXPERIMENTAL SET-UP}

\section{A. Geometry and measurements}

The experimental set-up is illustrated in Fig. 1. A ground plate is placed in an Eiffel type wind tunnel having a turbulent intensity less than $0.3 \%$. The homogeneity of the velocity over the $390 \mathrm{~mm} \times 400 \mathrm{~mm}$ section is $0.4 \%$. The wake is generated by the square-back geometry first used in the experiments of [23] as a simplified model to study ground vehicle aerodynamics. The total length of the body is $L=261.0 \mathrm{~mm}$, the height $H$ and width $W$ of the base are respectively $72.0 \mathrm{~mm}$ and
$97.2 \mathrm{~mm}$. The four supports are cylindrical with a diameter of $7.5 \mathrm{~mm}$. The blockage ratio is less than $5 \%$. The coordinate system is defined as $x$ in the streamwise direction, $z$ normal to the ground and $y$ forming a direct trihedral. The Reynolds number of the flow is defined as $\operatorname{Re}=U_{0} H / \nu$ where $U_{0}$ is the uniform flow velocity ranging from $3.7 \mathrm{~m} \cdot \mathrm{s}^{-1}$ to $33 \mathrm{~m} \cdot \mathrm{s}^{-1}$ and $\nu$ the air kinematic viscosity. The explored range of Reynolds numbers is thus comprised within $1.7 \times 10^{4}$ to $1.6 \times 10^{5}$.

The body is fixed on a turntable to allow side slip conditions with a yaw angle $\beta$ (see Fig. 1b). The rotation mechanism is driven by a displacement controlled by a Newport Motion Controller ESP301; the precision of the robot is better than $0.02^{\circ}$. Another displacement also controlled by the ESP301 allows adjustment of the ground clearance in the range $0<C<12.5 \mathrm{~mm}$. The precision is better than $10 \mu \mathrm{m}$. In the following experiments, the ground clearance is increased in steps of $250 \mu \mathrm{m}$.

The pressure on the body is measured at 21 locations at the body base (see blue dots in Fig. 1c). The taps are distributed symmetrically referring to the planes $y=0$ and $z=0$, the latter plane corresponding to the midheight of the geometry. The pressure is obtained using a ZOC22 pressure scanner and a GLE/SmartZOC-100 electronic for data acquisition using an ethernet connection to the PC. The high cut-off frequency of each transducer is larger than $250 \mathrm{~Hz}$. It is acquired at a sample rate of $500 \mathrm{~Hz}$ per channel, with an accuracy of $\pm 3.75 \mathrm{~Pa}$. The pressure scanner is located inside the model; it is denoted "P scan" in Figs. 1 $(a, b)$. It is linked to each tap with less than $100 \mathrm{~mm}$ of vinyl tubes to limit the filtering effect of the tubing. In that case, the high cut-off frequency falls to $150 \mathrm{~Hz}$. This device is connected to the electronic GLE/SmartZOC-100 using a connection cable going through one of the four cylindrical supports of the model so that, apart from these supports, nothing disturbs the underbody flow. The pressure coefficient is calculated as follows:

$$
C_{p}=\frac{p-p_{0}}{\frac{1}{2} \rho U_{0}^{2}}
$$

where $p_{0}$ is the static pressure in the tunnel just before the test section, the dynamic pressure $\frac{1}{2} \rho U_{0}^{2}$ is measured from a Pitot tube at the entrance of the test section.

In the following, the use of an asterisk for $a^{*}$ denotes the non-dimensional value of any quantity $a(x, y, z, t)$ made dimensionless by a combination of the height $H$ and the inlet velocity $U_{0}$.

\section{B. Free stream characterization}

In order to control the constant flow conditions, the ground plate (floor) is placed at $10 \mathrm{~mm}$ above the bottom side of the inlet (see Fig. 1a) and triggers the boundary layer at its leading edge, without any separation, $140 \mathrm{~mm}$ upstream of the fore-body. The boundary layer 
(a)

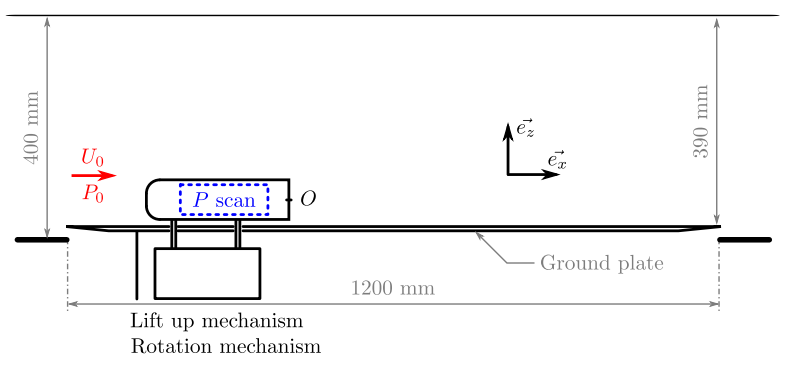

(b)

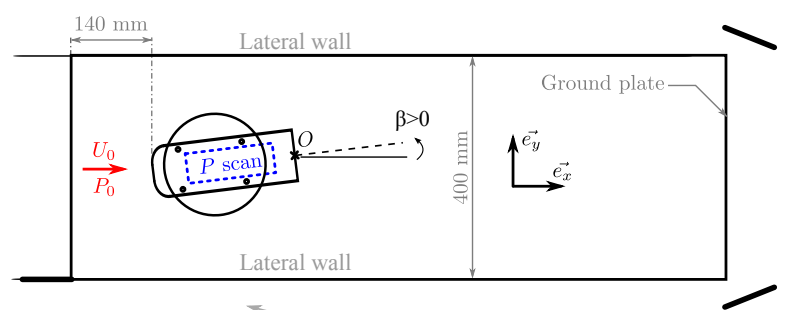

(c)

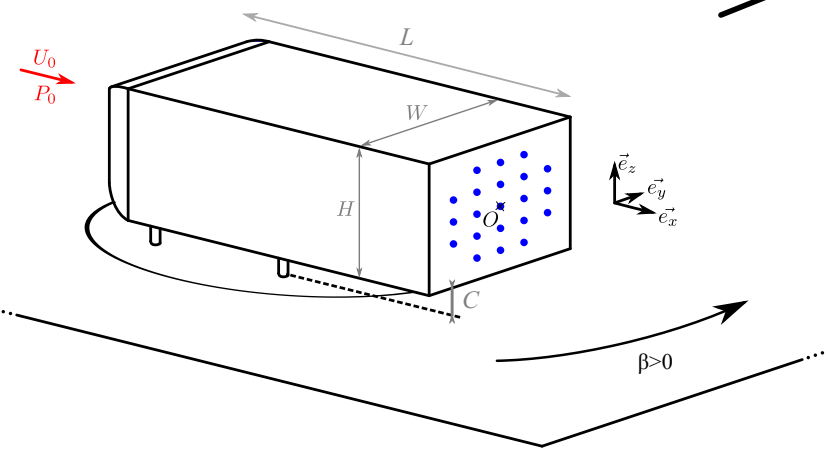

FIG. 1: Experimental setup: side view (a), top view (b) and perspective view (c); the point $\mathrm{O}$ at the centre of the body base sets the origin of the coordinate system. The blue dots locate the visible pressure taps; $\mathrm{P}$ scan refers to the pressure scanner. (b)

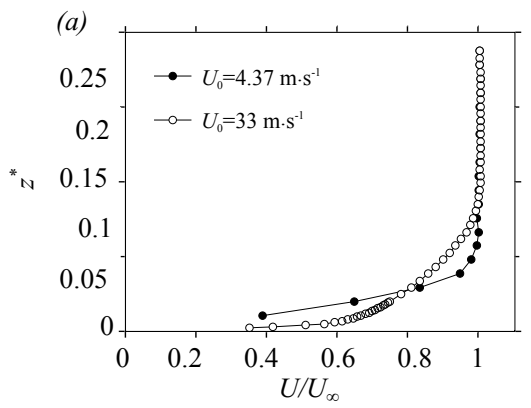

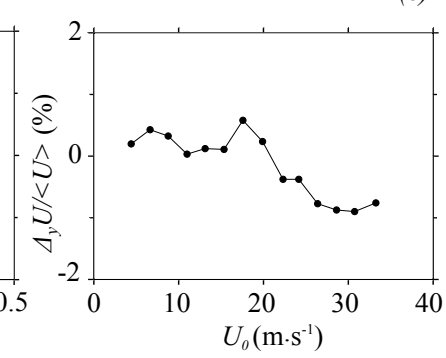

FIG. 2: Free stream characterization at the location of the Ahmed body, boundary layer profile on the floor(a), lateral velocity profile at $z^{*}=1 / 2(\mathrm{~b})$, right to left asymmetry (see text) of the lateral velocity profile vs. the main velocity (c). on the floor of the free stream at the body location (say $X=260 \mathrm{~mm}$ from the leading edge of the ground plate and without the body) is shown in Fig. 2 for the two extreme main flow velocities of the explored range. The boundary layer profile at the lowest velocity has a Reynolds number based on the development length $\operatorname{Re}_{X}=0.75 \times 10^{5}$ and suggests that it is laminar at the lowest velocity. At the largest velocity, $\operatorname{Re}_{X}=5.72 \times 10^{5}$ and the shape suggests a turbulent boundary layer. For both cases, the thickness never exceeds $\delta_{0.99}^{*}=0.1$. The laminar-turbulent transition occurs around $14 \mathrm{~m} \cdot \mathrm{s}^{-1}$, say $\operatorname{Re}_{X}=2.42 \times 10^{5}$. However, this transition has no meaning considering the flow around the Ahmed body whose presence produces pressure gradients at the ground wall that modifies drastically the boundary layer history compared to a development on the flat floor alone.

The spanwise spatial homogeneity is characterized through the velocity measurements in Fig. 2(b). Although the spatial inhomogeneity still remains below $0.4 \%$ in $r m s$ quantities, the streamwise velocity displays a small but significant shear whose sign depends upon the mean flow velocity. Figure $2(c)$ shows the difference between the mean velocity computed from the right hand side of the tunnel $(y>0)$ and the left hand side of the tunnel $(y<0)$. There is a velocity excess on the left hand side of the free stream of about $1 \%$ for mean flow velocities larger than $20 \mathrm{~m} \cdot \mathrm{s}^{-1}$. These symmetrical defects in the free stream have to be acknowledged considering their induced bias in the onset of a symmetry breaking instability.

\section{RESULTS}

\section{A. Global quantities for the near wake}

The pressure distribution at the base of the body has been shown to be a relevant indicator [19, 20, 24, 25] of the large scale wake topology. As in [25], we will use the instantaneous barycentre of the base pressure distribution, that we define as :

$$
\vec{r}_{b}\left(y_{b}, z_{b}\right)=\frac{\iint_{\text {Base }} \vec{r} C_{p}(\vec{r}) d y d z}{\iint_{\text {Base }} C_{p}(\vec{r}) d y d z},
$$

It should be noted that for a massively separated wake flow as in the present case, the pressure of the base is always lower than the static pressure of the free stream meaning that the denominator in Eq. 2 is non-zero and always negative. Figure $3(a, b)$ shows the two components of the base pressure barycentre. Its horizontal component in Fig. 3(a) clearly exhibits the bi-modal behavior. The two most probable horizontal positions observed at $y_{b}^{*} \simeq \pm 0.05$ are associated with the two deflected wakes fully characterized in [19]. They correspond to the two mirror $R S B$ modes breaking the reflectional symmetry with respect of the plane $y=0$ of the set-up. As it has already been shown in [19] the characteristic timescale to 
switch between these modes is 2 to 3 orders of magnitude larger than the natural timescale $T^{*}=1$ in non dimensional units, (i.e. $\left.T=\frac{H}{U_{0}}\right)$. For the remainder of this study, time series are low-pass filtered to focus on this long time dynamics, with a cut-off frequency $f_{c}^{*}=\frac{1}{250}$ (say $f_{c}=0.22 \mathrm{~Hz}$ at $U_{0}=4 \mathrm{~m} \cdot \mathrm{s}^{-1}$ to $f_{c}=1.83 \mathrm{~Hz}$ at $\left.U_{0}=33 \mathrm{~m} \cdot \mathrm{s}^{-1}\right)$. The filtered data are shown for the barycentre coordinates in Fig. 3(c).

The most probable positions of the pressure barycentre are extracted from probability density functions (PDFs) such as the one presented in Fig. 4. The statistics are performed over a duration fixed to $50 \mathrm{~s},\left(\tau^{*}=27500\right.$ at $U_{0}=33 \mathrm{~m} \cdot \mathrm{s}^{-1}$ and $\tau^{*}=3300$ at $\left.U_{0}=4 \mathrm{~m} \cdot \mathrm{s}^{-1}\right)$. The choice of recording duration is a compromise between an accurate estimation of the position of the most probable events and the high cost of time due to a parametric study involving changes in yaw angle, ground clearance and Reynolds number. As we can attest from Fig. 4, the most probable event positions are sufficiently well defined to investigate the parametric study of the stable branch solutions. Note that the chosen recording time that evolves in the range $\tau^{*} \sim 3000-27500$ leads unavoidably to weakly converged PDFs because of the long time dynamics associated with the $R S B$ modes having a characteristic timescale in the range $\tau^{*} \sim 100-1000$ [19]. Hence, the weak convergence does not allow an accurate estimate of the value of the corresponding probability density.

Another global quantity of interest is the base suction $-C_{p b}$, where :

$$
C_{p b}=\frac{1}{S} \iint_{\text {Base }} C_{p}(\vec{r}) d y d z .
$$

The base suction is directly related to the form drag (see [26], [27] and references therein), the larger the base suction, the larger the form drag. Note that the discrete pressure taps distribution at the base (Fig. 1c) will not lead to an exact measurement of the base suction but rather to a drag indicator.

B. Wake mode sensitivity to a yaw angle $\beta$ and ground clearance $C^{*}$

Figure 5 shows the PDFs of the statistical variable $y_{b}^{*}$, for 10 sets of experiments performed for the same Reynolds number Re $=146800$. The grey levels of the PDFs allow the most probable positions of the barycentre, which contains the main information, to be located. For each set, the ground clearance $C^{*}$ is fixed and the yaw angle is varied within the range $-1^{\circ}<\beta<+1^{\circ}$ in step of $0.1^{\circ}$. By looking at the PDFs at the top of Fig. 5 obtained for the ground clearance $C^{*}=0.153$, we notice that two distinguished most probable positions, symmetrically located at $y_{b}^{*+} \simeq 0.05$ and $y_{b}^{*-} \simeq-0.05$ are observable. These two positions are associated with the two stable mirror states and the values of $y_{b}^{\star \pm}$ do not depend upon the yaw angle. When the ground clearance
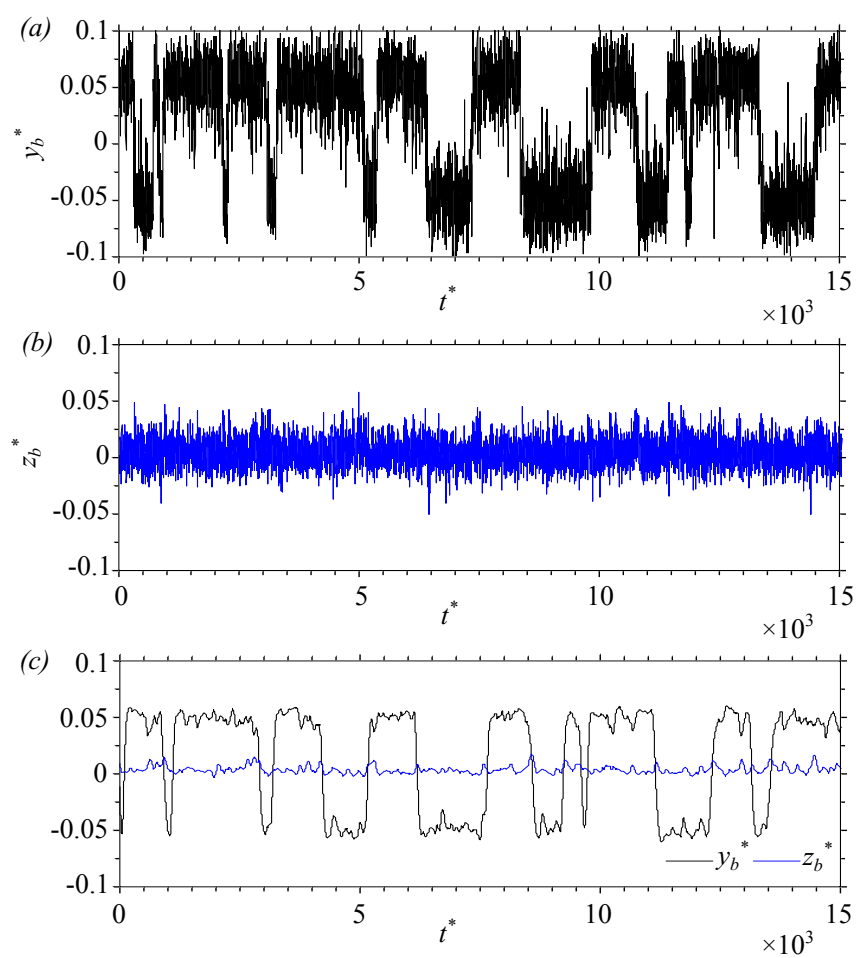

FIG. 3: Time series of the base pressure barycenter coordinates $\left(y_{b}^{*}, z_{b}^{*}\right), y_{b}^{*}(\mathrm{a}), z_{b}^{*}(\mathrm{~b})$ and filtered in (c).

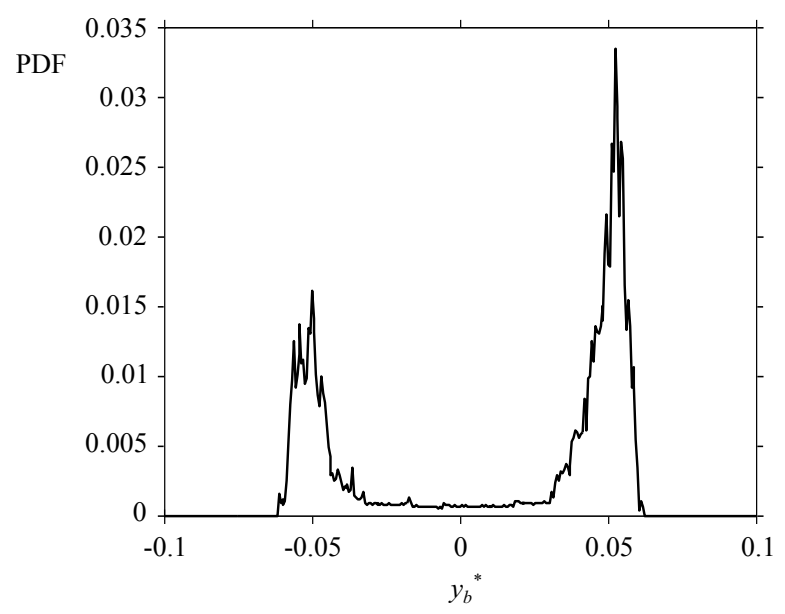

FIG. 4: Probability density function (PDF) of the horizontal coordinate $y_{b}^{*}$ of the base pressure barycenter computed from the time serie in Fig. 3(c).

is decreased to $C^{*}=0.09$, similar observations can be made except that now the two positions are not symmetrically located anymore. It emphasizes the sensitivity to symmetry defects in the setup, especially related to the non-uniformity of the incoming flow as described in Fig. 2.

For slightly smaller $C^{*}$, the two states become hardly distinguishable and instead, a continuous change in the pressure barycentre coordinate is observed.

The role of the underbody flow as a function of the 


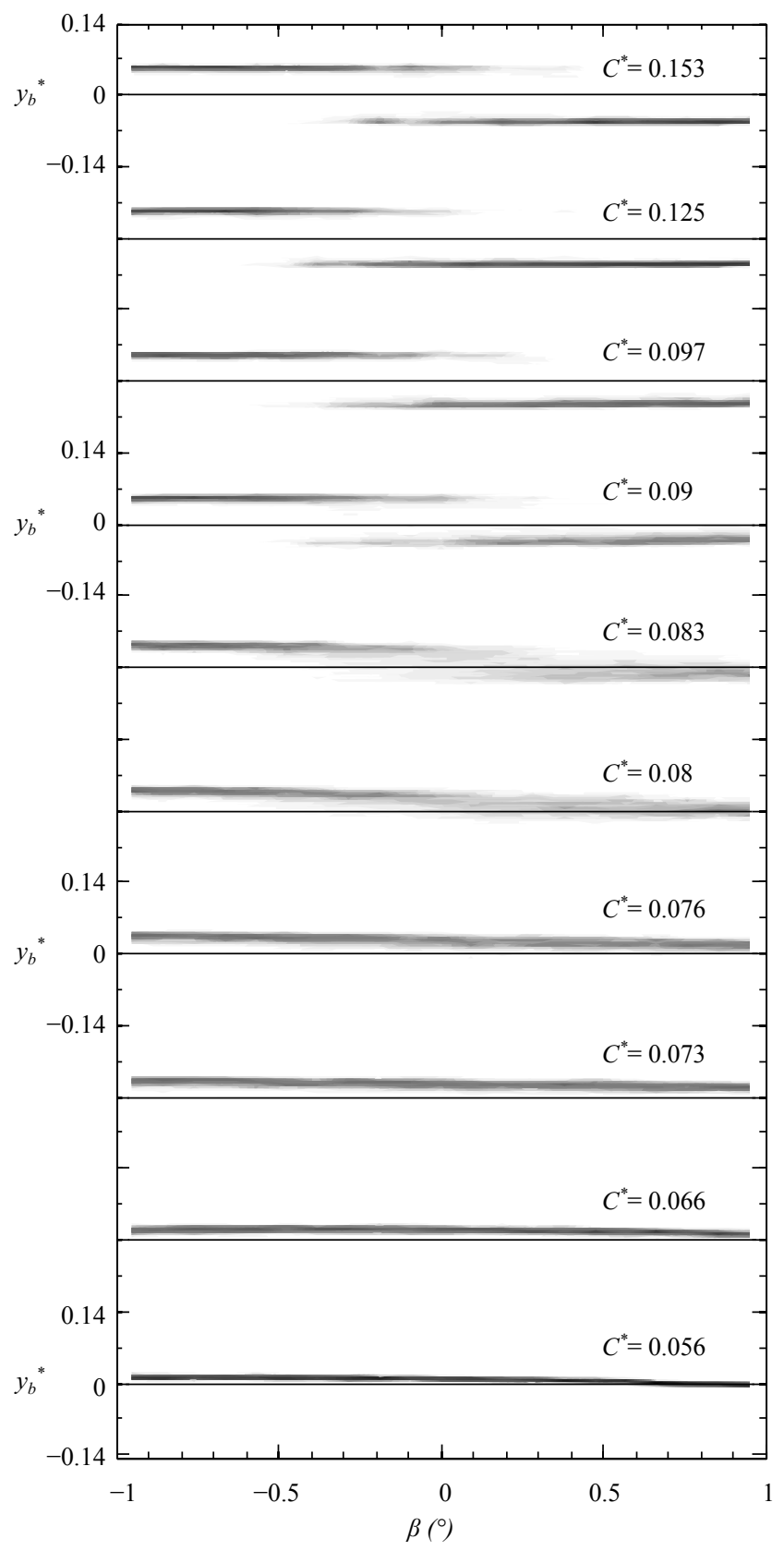

FIG. 5: Evolution of the probability density function, $\operatorname{PDF}\left(y_{b}^{*}\right)$ in grey levels (the darker, the larger the PDF) for $U_{0}=30.58 \mathrm{~m} \cdot \mathrm{s}^{-1}$ with the yaw angle $\beta$ and for different ground clearances $C^{*}$.

ground clearance is now investigated. Velocity profiles are then measured $2 \mathrm{~mm}$ behind the body using a classical hot wire probe, in the mid plane $y=0$ for different ground clearances $C^{*}$. They are all presented in Fig. 6 as $z / C$ vs. the velocity with a vertical shift for clarity. For large ground clearances, $C^{*}>0.04$, velocity profiles are well established and present a relatively parabolic shape. The velocity fluctuations are maximum (Fig. 8b) on the top side of the profiles which corresponds to the free mixing layer, and on the bottom side that corresponds to
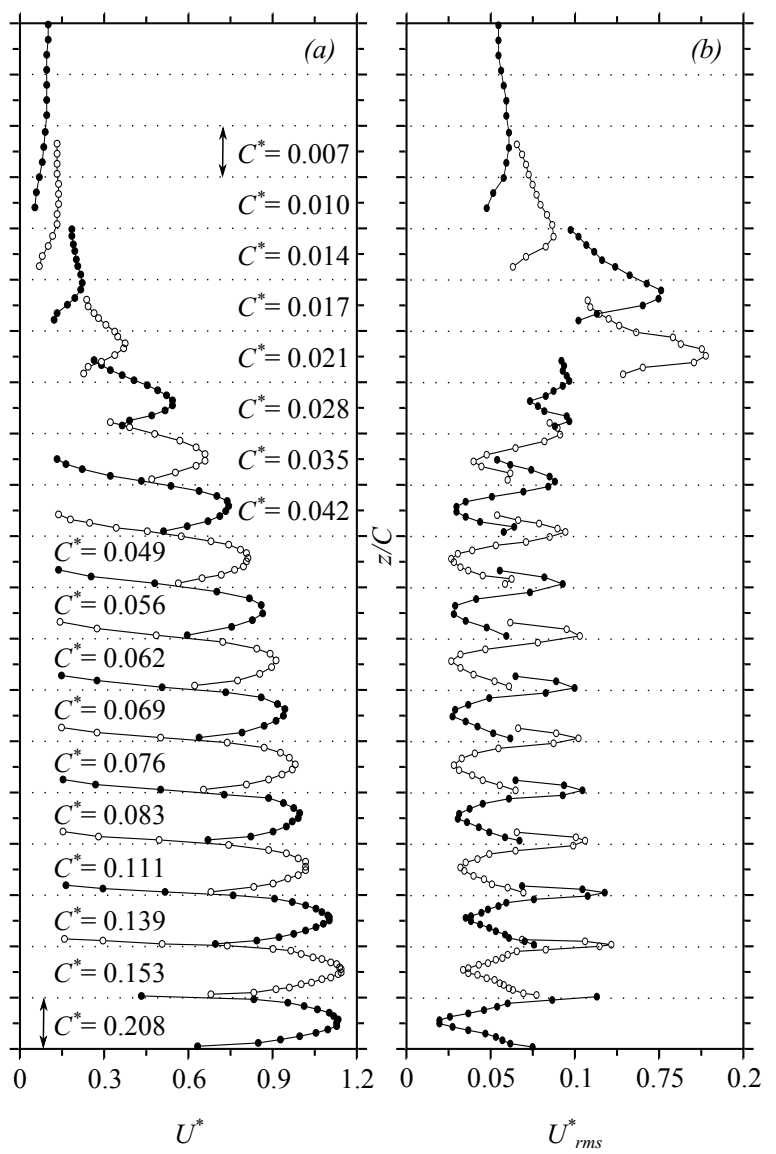

FIG. 6: Characterization of the underbody flow using hot wire anemometer measurements, $2 \mathrm{~mm}$ behind the body in the $y=0$ plane. The hot wire is oriented to be sensitive to the longitudinal velocity $u(t)$. (a) $: z / C$ vs. the time averaged non-dimensional velocity $U^{*}=\overline{u^{*}}$. (b) Fluctuating velocity defined as $U_{r m s}^{*}=\sqrt{\overline{\left(u^{*}-U^{*}\right)^{2}}}$.

the boundary layer on the floor. For ground clearances smaller than $C^{*}<0.04$, there is no established underbody flow and a transition with high velocity fluctuations is observed for $C^{*}=0.021$.

To study the two stable solutions of the bifurcated states, we chose to explore the wake positions for the two yaw angles $\beta=-0.4^{\circ}$ in Fig. $7(a)$ and $\beta=+0.4^{\circ}$ in Fig. $7(b)$. The negative (resp. positive) yaw angle allows exploration of the branch $y_{b}^{*+}$ (resp. $y_{b}^{*-}$ ) corresponding to the positive (resp. negative) part of $\operatorname{PDF}\left(y_{b}^{*}\right)$. As can be seen, the system explores preferentially the $y^{*+}$ branch near the bifurcation point which is an evidence for an imperfect bifurcation. In addition, we never noticed any hysteretic effect by working with increasing or decreasing control parameters, which reinforces the supercritical character of the bifurcation. For both PDF branches, the position of the local maxima are extracted (plotted as white lines in Fig. $7 a$ and $b$ ). This technique of extraction will be repeated in the following to study the Reynolds number effect on the bifurcation diagram. 

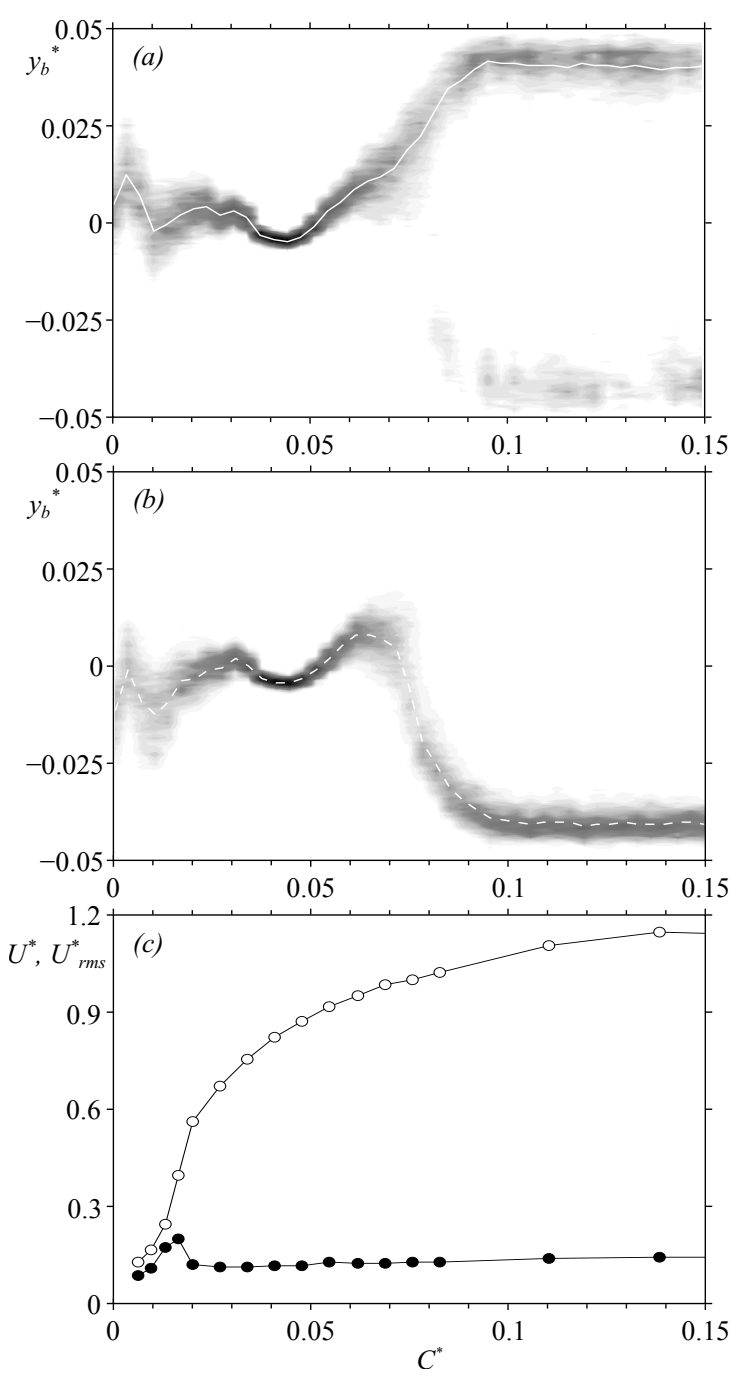

FIG. 7: Probability density functions, $\operatorname{PDF}\left(y_{b}^{*}\right)$ in grey levels for $U_{0}=30.58 \mathrm{~m} \cdot \mathrm{s}^{-1}$ vs. the ground clearance $C^{*}$, for $(a)$ with the yaw angle $\beta=-0.4^{\circ}$ and $(b)$ with a yaw angle $\beta=$ $+0.4^{\circ}$. The white lines represent the most probable position and correspond in ( $a$, continuous line) to the stable branch $y_{b}^{*+}$ and in ( $b$, dashed line) to the other stable branch $y_{b}^{*-}$. In $(c)$ are reported the maximum of the profiles measured in Fig. 6, mean velocity $U^{*}$ (empty circles) and fluctuation $U_{r m s}^{*}$ (black filled circles).

Figure $7(c)$ displays the maximum values of the profiles measured in Fig. 6. It is clear from these results that the underbody flow is already well established, about $U_{0}$ at the pitchfork point of the bifurcation observed around $C^{*} \sim 0.075$. Hence, the large velocity fluctuations observed at $C^{*}=0.021$ occurs at a too small ground clearance to be associated with the instability threshold of the $R S B$ states.

\section{Bifurcation threshold with Reynolds number}

Nine experiments with different Reynolds numbers have been conducted with exactly the same protocol as for the previous experiment. All of the bifurcation diagrams obtained by superimposing the branch $y^{*+}$ and $y^{*-}$ are shown in Fig. 8. We can see clear imperfect pitchfork bifurcations due to some symmetry defects before and at the bifurcation point. As said above, these imperfections are likely to be explained by the free stream properties characterized in Fig. 2. The bifurcation point, defines as the crossing point between the two branches, appears to depend on the Reynolds number, and its dependency is shown in Fig. 9. The uncertainty about $C_{c}^{*}$ is estimated to be \pm 0.0025 reflecting the measurements displayed in Fig. 8(a).

The critical ground clearance $C_{c}^{*}$ is a slow decreasing function of the critical Reynolds number $R e_{c}$, similar to the power law $\operatorname{Re}_{c}^{-1 / 6}$ in the range of our experiments.

\section{Toward a stabilization using passive control}

We now investigate the possibility for the existence of an unstable branch solution, corresponding to the prolongation of the symmetric state after the bifurcation point. The idea is to apply the passive control technique first introduced by [28] to stabilize the Kármán instability in the laminar wake of a circular cylinder. It has been shown for the present configuration in [24] to efficiently eliminate the bi-modal behavior of the turbulent wake. It consists in a steady disturbance technique by introducing inside the recirculating bubble a vertical cylinder, having the same height as the body with a diameter $d^{*}=0.083$. We placed the control cylinder at the best location obtained in [24], say $y^{*}=0$ and $x^{*}=0.52$. Two thin rods, as depicted in Fig.10(b), support the control cylinder. In order to characterize the effect of the control cylinder only, a bifurcation diagram has been performed with the body and the two thin rods as a function of the ground clearance. It is shown in Fig.10(a). Because of the thickness of the rod under the body, the ground clearance is limited for low values to $C^{*}=0.035$. The observed bifurcation, very similar to the one obtained without the rods (Fig.7), attests from the neutrality of the supporting system. Furthermore, it has to be mentioned that the bifurcation in Fig.10(a) is obtained for only one yaw angle. It has been especially adjusted in order to have the best equiprobable exploration of the two states at the largest ground clearance. In this condition, we see that both branches are randomly explored after the bifurcation point, leading to the bistable dynamics of the wake.

When the control cylinder is placed between the two rods, the bifurcation diagram before the pitchfork point remains unaltered compared to that of the reference, while after the pitchfork point the two branches are replaced by a continuum of uniform density probability 


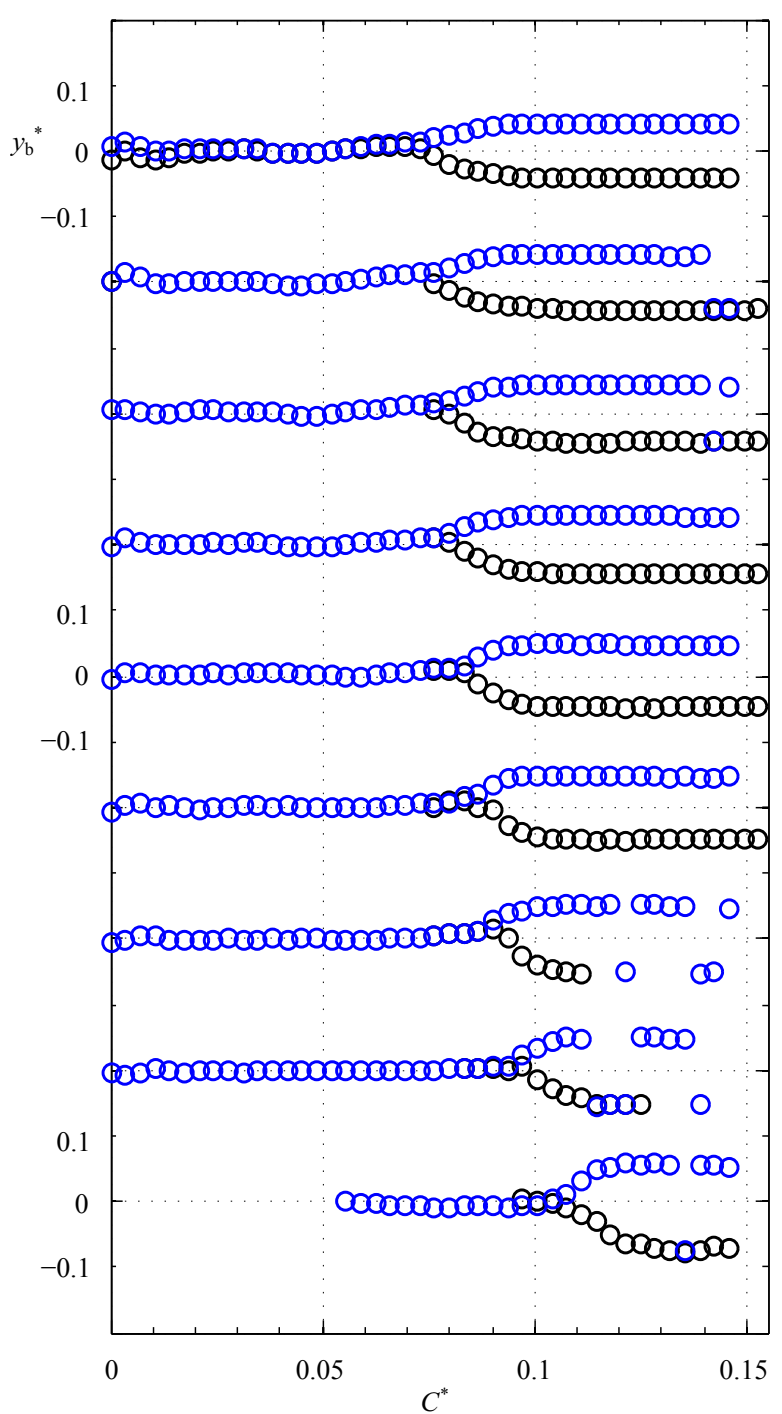

FIG. 8: Bifurcation diagrams vs. the ground clearance obtained with different Reynolds numbers, from bottom to top : $\mathrm{Re}=1.78 \times 10^{4}, 2.89 \times 10^{4}, 3.95 \times 10^{4}, 5.05 \times 10^{4}, 6.1 \times 10^{4}, 8.27 \times$ $10^{4}, 1.04 \times 10^{5}, 1.32 \times 10^{5}, 1.6 \times 10^{5}$. For each Reynolds number, the branches are extracted from the most probable position of the barycentre obtained from $\beta=-0.4^{\circ}$ (blue circles) and $\beta=+0.4^{\circ}$ (white circles) as in Fig. $7(a)$ and $(b)$.

over values of $y^{\star}$ included within $y_{b}^{\star \pm}$, yet excluding $y_{b}^{\star \pm}$. At this point it is difficult to argue whether the system is actually stabilized since there is no most probable position around $y^{*}=0$. However the system is definitely not bi-modal anymore. This simple experiment is promising since passive control can be considerably improved using active control. We are confident that the latter will be the good strategy to stabilize the wake.

Although the suppression of the lateral force can be achieved by stabilizing the flow, it remains interesting to look at the effect of a stabilization on the base suction and hence the drag. The base suction as defined in Eq.3 is shown in Fig.11. The dashed line refers to the reference case, it displays large variations of the base suction

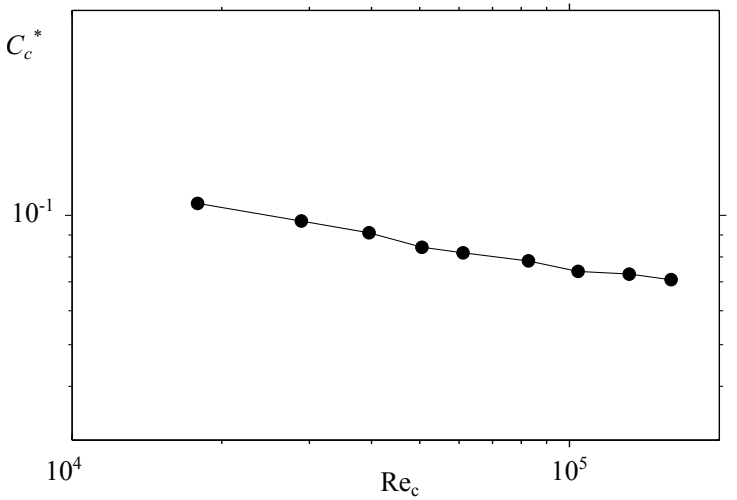

FIG. 9: Threshold of the bifurcation, critical ground clearance vs. critical Reynolds number.

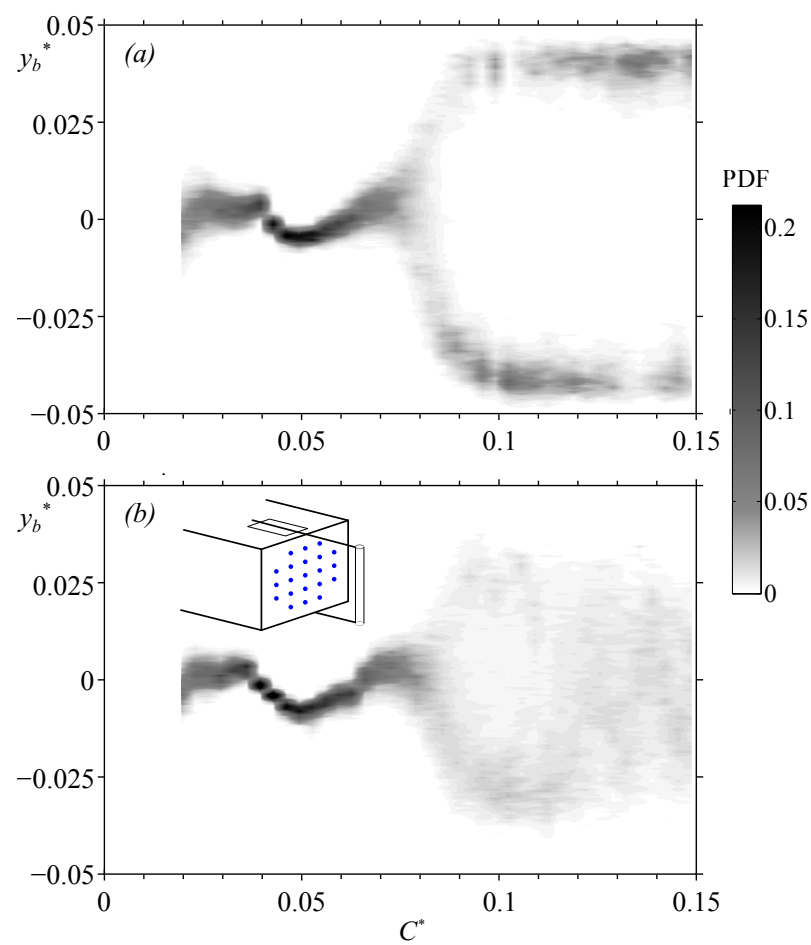

FIG. 10: Probability density functions, $\operatorname{PDF}\left(y_{b}^{*}\right)$ in grey levels for $U_{0}=30.58 \mathrm{~m} \cdot \mathrm{s}^{-1}$ vs. the ground clearance $C^{*}$, for $(a)$ with the yaw angle $\beta=-0.4^{\circ}$ and $(b)$ with a yaw angle $\beta=$ $+0.4^{\circ}$. The white lines represent the most probable position and correspond in ( $a$, continuous line) to the stable branch $y_{b}^{*+}$ and in ( $b$, dashed line) to the other stable branch $y_{b}^{*-}$.

coefficient due to the development of the underbody flow as already described in [20] and characterized in Fig. 6. However, the abrupt change observed for $C^{*} \sim 0.09$ is clearly ascribed to the pitchfork point, indicating that the presence of the $R S B$ modes are creating additional base suction and then drag. By stabilizing the wake, one would expect the base suction coefficient to decrease in the continuity of its evolution before the pitchfork point. It is actually what is observed when the control cylinder 


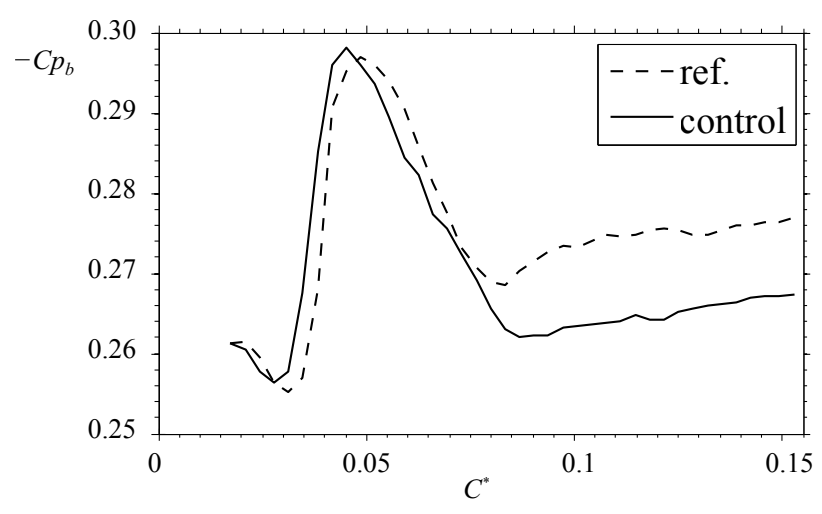

FIG. 11: Base pressure suction vs. the ground clearance for (dashed line) the reference case of Fig. 10(a) and for (continuous line) the controlled case of Fig. 10(b).

is added, the base suction follows the same variations as for the reference case. After the instability threshold, a significant reduction is observed. This passive control experiment is encouraging as a new strategy for drag reduction of 3D turbulent wakes.

\section{E. Flow visualizations}

We turn now to an illustrative experiment of flow visualization realized in a water tunnel. The model is a 1:2.66 scale of the one used above. The test section of the tunnel is $80 \times 150 \mathrm{~mm}$ and the flow speed $6 \mathrm{~m} \cdot \mathrm{s}^{-1}$. The Reynolds number is 216000 , and the ground clearance is set to $C^{*}=0.1$. The yaw angle is accurately adjusted to obtain the bistable dynamics of the two $R S B$ states. We present in Fig. 12, some pictures extracted from a long time movie showing the random switching between the $y_{b}^{+}$stable solution shown in (a) and the $y_{b}^{-}$stable solution shown in (b). The two wake states present a mirror symmetry, with an intense circular recirculation clearly visible on the right hand side in Fig. 12(a) and symmetrically on the left hand side in Fig. 12(b). The low pressure barycentre position is on the same side as this intense recirculation. These flow visualizations are in total agreements with the velocity field measurements of the $R S B$ modes in [19]. When a vertical control cylinder is added as in paragraph III D, a symmetric wake is observed without any intense recirculations.

\section{CONCLUSIVE REMARKS}

The wake of a square-back body at the proximity of a ground wall undergoes a pitchfork bifurcation in the turbulent regime from a symmetric turbulent wake toward two asymmetric turbulent wakes. The phase diagram in Fig. 9 indicates that the critical ground clearance is a slow decreasing function of the critical Reynolds number. Thus, the bifurcation is also observable for a given
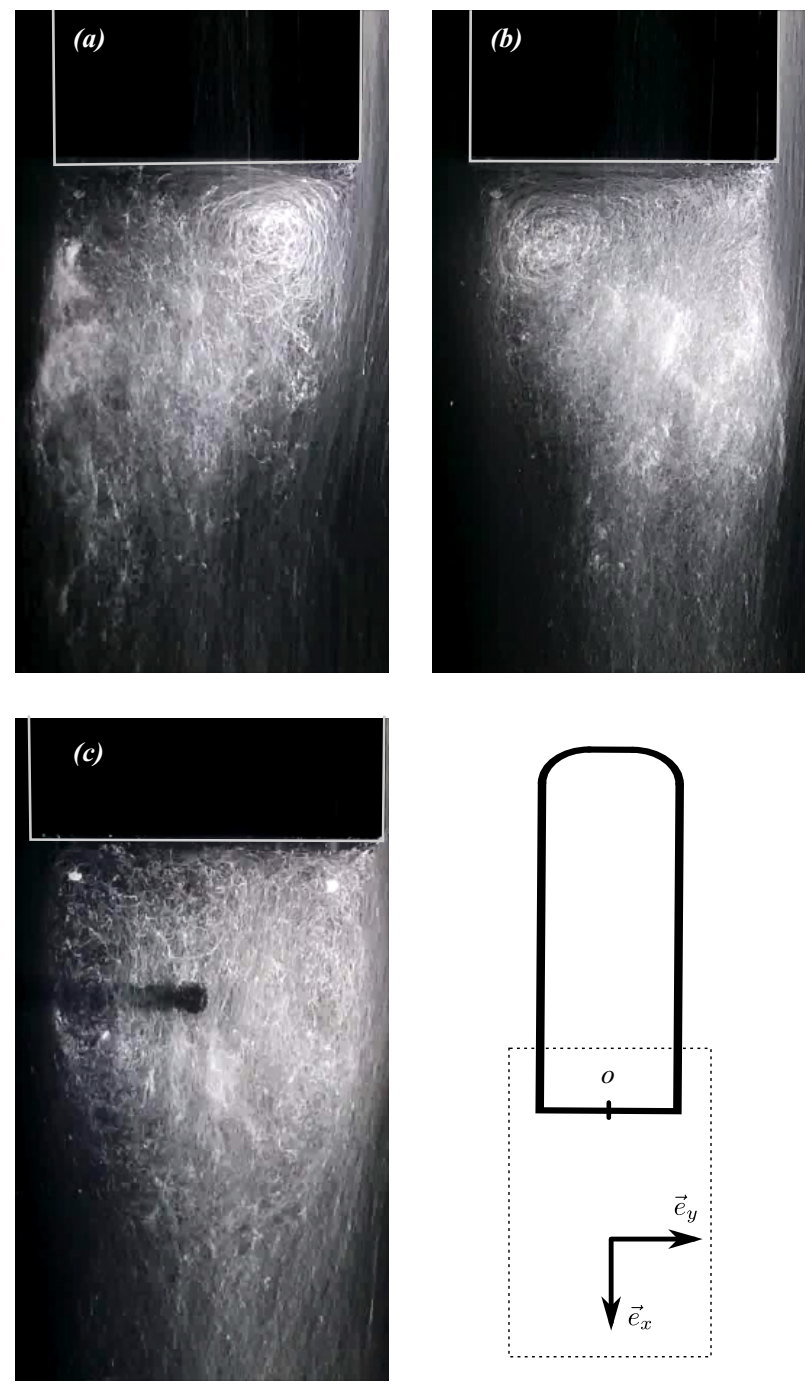

FIG. 12: Flow visualization performed in a water tunnel using bubbles appearing white against a black background. The flow Reynolds number is $R e=216000$ at a ground clearance $C^{*}=0.1$. The drawing shows the visualization area depicted by a dashed rectangle at the rear of the Ahmed body. The obturation time of the camera is $\tau^{*}=3$, say 3 times larger than the convective time $H / U_{0}$ and captures the dynamics at this timescale. In (a), $R S B$ mode of the branch $y_{b}^{+}$, in (b) its mirror counterpart: the $R S B$ mode of the branch $y_{b}^{-}$. Wake controlled with a vertical control cylinder (diameter $\left.d^{*}=0.107\right)$.

ground clearance as the Reynolds number is increased. The smaller the ground clearance the larger the Reynolds number for the transition. At the threshold of the instability (pitchfork point), the underbody flow magnitude is approximately $U_{0}$ (see Fig. 7c) indicating that the bifurcation is not related to a flow transition in the ground clearance. Following the idea of Grandemange et al. [20], the crucial ingredient for the symmetry breaking is the ratio between the separating distances of the vortex sheets produced by the separation at the rectangular base (it is also the result of the stability analysis by 
Marquet and Larsson [22] for a rectangular plate facing a uniform flow in the laminar regime). The inviscid condition at the ground wall, that can be justified for large Re flow, is equivalent to a mirror flow. In the absence of the underbody flow, the top vortex sheet emerging from the square-back body is then facing its symmetric counter part at a distance that is the double of the body height, $2 H$. In the case of an underbody flow, a second vortex sheet emerges from the bottom trailing edge of the body and the separating distance with the top vortex sheet is now $H$. For the primary case, the aspect ratio is twice the second case and does not allow the symmetry breaking instability at the given width $W$ of the base [20]. Hence the large Reynolds number bifurcation appears to be related to a stabilizing geometrical parameter. One may wonder if an equivalent explanation can be tested on the transition obtained with the von-Kármán swirling flow geometry.

There are some interesting applications for flow control of 3D turbulent wakes. For instance, the pioneering technique of Strykowski and Sreenivasan [29] to stabilize the periodic laminar Kármán instability toward the steady state is also efficient to stabilize the turbulent $R S B$ mode toward a symmetric wake mode. In addition to the flow studied here, it is known that the turbulent wake of axisymmetric bodies develops symmetry breaking modes as well $[25,30]$. Thus the stabilization toward the symmetric unstable mode might be a relevant strategy for drag and lateral force reduction in many applications with potentially a low energetic cost and offers promising future development.

\section{Acknowledgments}

The authors are grateful to Romain Monchaux for useful discussions about the von-Kármán swirling flow and thankful to John Wrightson for his critical reading of the manuscript. This work benefited from the financial support of the Labex LaSIPS in the framework of the TURBFORK project.
[1] U. Frisch, Turbulence (Cambridge University Press, 1996).

[2] H. Schlichting and K. Gersten, Boundary layer theory (Springer Verlag, 2000).

[3] P. Bearman, Journal of Fluid Mechanics 37, 577 (1969).

[4] E. Achenbach and E. Heinecke, Journal of Fluid Mechanics 109, 239 (1981).

[5] G. Schewe, Journal of Fluid Mechanics 133, 265 (1983).

[6] G. Schewe, Journal of Fluid Mechanics 172, 33 (1986).

[7] C. Farell and J. Blessmann, Journal of Fluid Mechanics 136, 375 (1983).

[8] J. J. Miau, H. W. Tsai, Y. J. Lin, J. K. Tu, C. H. Fang, and M. C. Chen, Experiments in Fluids 51, 949 (2011).

[9] Y.-J. Lin, J.-J. Miau, J.-K. Tu, and H.-W. Tsai, AIAA Journal 49, 1857 (2011).

[10] O. Cadot, A. Desai, S. Mittal, S. Saxena, and B. Chandra, Physics of Fluids 27, 014101 (2015).

[11] F. Ravelet, L. Marie, A. Chiffaudel, and F. Daviaud, Physical Review Letters 93, 164501 (2004).

[12] A. de la Torre and J. Burguete, Physical Review Letters 99, 054101 (2007)

[13] F. Ravelet and F. Chiffaudel, A.and Daviaud, Journal of Fluids Mechanics 601, 339 (2008).

[14] P.-P. Cortet, A. Chiffaudel, F. Daviaud, and B. Dubrulle, Physical Review Letters 105, 214501 (2010).

[15] P.-P. Cortet, E. Herbert, A. Chiffaudel, F. Daviaud, B. Dubrulle, and V. Padilla, Journal of Statistical Mechanics-Theory and Experiment P07012 (2011).

[16] C. Nore, L. Tuckerman, O. Daube, and S. Xin, Journal of Fluid Mechanics 477, 51 (2003).
[17] C. Nore, L. Witkowski, E. Foucault, J. Pecheux, O. Daube, and P. Le Quere, Physics of Fluids 18, 054102 (2006).

[18] M. Grandemange, M. Gohlke, and O. Cadot, Physical Review E 86, 035302 (2012).

[19] M. Grandemange, M. Gohlke, and O. Cadot, Journal of Fluid Mechanics 722, 51 (2013).

[20] M. Grandemange, M. Gohlke, and O. Cadot, Physics of Fluids 25, 095103 (2013).

[21] B. Pier, Journal of Fluid Mechanics 603, 39 (2008).

[22] O. Marquet and M. Larsson, European Journal of Mechanics B/Fluids 49, 400 (2014).

[23] S. Ahmed, G. Ramm, and G. Faitin, SAE Technical Paper Series 840300 (1984).

[24] M. Grandemange, M. Gohlke, and O. Cadot, Journal of Fluid Mechanics 752, 439 (2014).

[25] G. Rigas, A. Oxlade, A. Morgans, and J. Morrison, Journal of Fluid Mechanics 755, 159 (2014).

[26] A. Roshko, Journal of Wind Engineering and Industrial Aerodynamics 49, 79 (1993).

[27] C. Apelt and G. West, Journal of Fluid Mechanics 71, 145 (1975).

[28] P. Strykowski and K. Sreenivasan, Journal of Fluid Mechanics 218, 71 (1990).

[29] P. Strykowski and K. Sreenivasan, in AIAA Shear Flow Control Conference (1985).

[30] M. Grandemange, M. Gohlke, and O. Cadot, Experiments in fluids 55, 1 (2014). 